\title{
Amelioration of the DSS-induced colitis in mice by pretreatment with 4,4'-diaponeurosporene-producing Bacillus subtilis
}

\author{
YUCHAO JING, HAOFEI LIU, WENWEN XU and QIAN YANG \\ College of Veterinary Medicine, Nanjing Agricultural University, Nanjing, Jiangsu 210095, P.R. China
}

Received May 9, 2017; Accepted October 10, 2017

DOI: $10.3892 /$ etm.2017.5282

\begin{abstract}
Inflammatory bowel disease (IBD) is a chronically relapsing inflammatory disorder of the gastrointestinal tract. Current IBD treatments have poor tolerability and insufficient therapeutic efficacy, thus, alternative therapeutic approaches are required. Recently, a number of dietary supplements have emerged as promising interventions. In the present study oral administration of a carotenoid (4,4'-diaponeurosporene)-prod ucing Bacillus subtilis markedly ameliorated dextran sulfate sodium salt-induced mouse colitis, as demonstrated by a reduction in weight loss and the severity of bleeding, which indicated that 4,4'-diaponeurosporene may have beneficial effects on treatments for colitis. This preliminary study indicated that 4,4'-diaponeurosporene may function synergistically with probiotics to provide a novel and effective strategy to prevent colitis.
\end{abstract}

\section{Introduction}

Inflammatory bowel disease (IBD) in humans, including Crohn's disease and ulcerative colitis, is a complex chronic inflammatory disorder (1). The incidence of these diseases varies widely between different countries, but overall has increased greatly in recent years, making IBD a major public health problem now (2). Though its etiopathogenesis is ambiguous, there exist a growing awareness that oxidative stress and the resulting inflammation play an important role in the development of IBD (3-6).

Current IBD treatments include aminosalicylates, corticosteroids, inhibitors of tumor necrosis factor- $\alpha$ (TNF- $\alpha$ ), antibiotics and immunosuppressants (7). However, these agents have poor tolerability and insufficient therapeutic efficacy; therefore, the need for alternative therapeutic approaches is increasing $(8,9)$. It is now clear that probiotic intervention

Correspondence to: Professor Qian Yang, College of Veterinary Medicine, Nanjing Agricultural University, 6 Tong Wei Road, Nanjing, Jiangsu 210095, P.R. China

E-mail: zxbyq@njau.edu.cn

Key words: colitis, 4,4'-diaponeurosporene, Bacillus subtilis, probiotics is able to prevent pouchitis and has found to be effective in inducing and maintaining remission in ulcerative colitis, although reports might be conflicting, as they involve different mixtures of probiotic strains, different protocol designs, various doses, different read-outs and different clinical settings or patient types $(10,11)$. The Gram-positive spore-forming probiotic, Bacillus subtilis (B. subtilis), has been well demonstrated to have probiotic potential $(12,13)$. Spore-forming bacteria may offer many interesting advantages compared to non-sporeformers, as they are heat-stable, resistant to low $\mathrm{pH}$ and other deleterious conditions such as gastric and bile secretions in the intestinal environment. Moreover, spores allow long term storage of preparations without refrigeration or need for encapsulation processes (14). Notably, a number of studies have indicated the anti-inflammatory function of some B. subtilis strains in $\operatorname{IBD}(15,16)$.

Carotenoids, a subfamily of the isoprenoids containing more than 700 members, are currently used for food colorants and nutritional supplements (17). Carotenoids can act as antioxidants with the potential to remove free radicals, either by a direct reaction with radicals, resulting in the formation of harmless products, or by disrupting radical chain reactions, avoiding further damage of cellular compounds, such as membrane lipids. It was noted that patients with ulcerative colitis have extremely low concentrations of serum and mucosa carotenoids (such as lutein, zeaxanthin, $\alpha$ - and $\beta$-carotene) as compared to that of healthy individuals $(18,19)$. Moreover, several studies have showed that dietary carotenoids inhibited colitis and colitis-associated colon carcinogenesis in mice (20-22).

In previous study, we had constructed a carotenoid (4,4'-diap oneurosporene)-producing B. subtilis (B.s-Dia) by transforming a plasmid which containing carotenoid synthase gene, $\mathrm{crm}$, and carotenoid dehydrogenase gene, crtn, into B. subtilis strain WB800 (23). The aim of this study was to address the protective effect of carotenoid-producing $B$. subtilis on murine experimental colitis. Our results indicated that oral administration of B.s-Dia had an improved positive effect on colon histopathological changes and length in mice dextran sodium sulfate (DSS)-induced colitis.

\section{Materials and methods}

Mice. C57BL/6 mice (23-24 g), 8 weeks old, were purchased from the Animal Research Center of Yangzhou University 
A

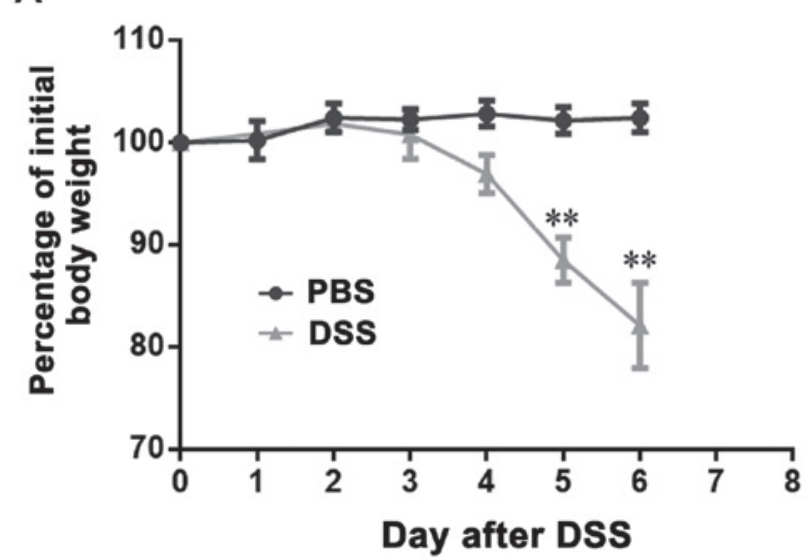

B

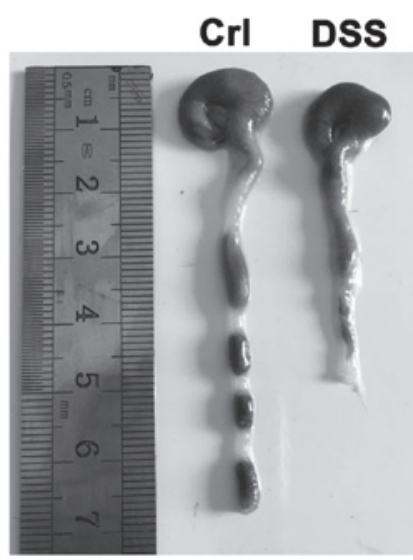

Figure 1. DSS-induced colitis model. (A) Mice were administered 5\% DSS in the drinking fluid for 6 days and weighed every day. (B) The colon was excised between the ileocaecal junction and the proximal rectum, close to its passage under the pelvisternum. The colon was placed on a non-absorbent surface and its length was measured with a ruler, in such a manner that the organ was not stretched. One representative of three similar independent experiments is shown. Asterisks indicate statistical significance via the one-way ANOVA test. ${ }^{* *} \mathrm{P}<0.01$ vs. the PBS-treated group. DSS, dextran sodium sulfate; Crl, control.

(Jiangsu, China). The mice were maintained under specific pathogen-free conditions for at least 1 week before use. The animal studies were approved by the Institutional Animal Care and Use Committee of Nanjing Agricultural University and followed National Institutes of Health guidelines for the performance of animal experiments.

Bacterial strains, animals. B.s-Dia was constructed as previously described. B.s or B.s-Dia were grown in Luria-Bertani (LB) broth (10 g tryptone, $5 \mathrm{~g}$ yeast extract, and $5 \mathrm{~g} \mathrm{NaCl} / \mathrm{l})$ containing $50 \mu \mathrm{g} / \mathrm{ml}$ kanamycin at $37^{\circ} \mathrm{C}$.

Induction of experimental colitis. To inducing colitis, DSS (molecular weight 5,000; MP Biomedicals Inc., Eschwege, Germany) was added into mouse drinking water at the concentration of $5 \%(\mathrm{w} / \mathrm{v})$ for 6 or 7 days. And mice were randomly equally divided into four groups ( $n=7$ in each) as follows: Control group was just given PBS by gavage each day for 7 days and no DSS was added in drinking water. DSS group was also given PBS by gavage once a day for 7 days but followed by DSS added drinking water every day for successively 7 days. B.s+DSS or B.s-Dia+DSS group was given $1 \times 10^{9}$ cfu B. subtilis Wb800 or B.s-Dia once a day for 7 days and followed by DSS added drinking water every day for successively 7 days. And the dose of B.s-Dia was selected based on the results of previous studies (24-27), and that a dose of $1 \times 109 \mathrm{cfu} / \mathrm{ml}$ also has been demonstrated to significantly alleviate colitis (28).

Assessment of mouse weight and colon length. Mouse weight was measured daily from day 0 to 7 at 9:30 a.m. every day and expressed as the relative change from day 0 . The colon was isolated immediately after the last weight check. The colon was excised between the ileocaecal junction and the proximal rectum, close to its passage under the pelvisternum. The colon was placed on a non-absorbent surface and its length was measured with a ruler, in such a manner that the organ was not stretched. Mouse weight and colon length were determined as described previously $(29,30)$.
Histological observation and scoring. The morphological evaluation of colon in each group was performed using hematoxylin and eosin (H\&E) staining. The colons were fixed in $4 \%$ paraformaldehyde, embedded in paraffin and sliced into sections of $5 \mu \mathrm{m}$ thickness. After H\&E staining, histological analysis was performed in a blind fashion. Colitis was assessed with tissue sections and was scored in a blinded experimental set-up according to a standard scoring system: 0, no thickening of colonic tissues and no inflammation (infiltration of lymphocytes); 1 , mild thickening of tissues but no inflammation; 2, mild thickening of tissues and mild inflammation; 3 , severe thickening and severe inflammation (31).

Statistical analysis. Results were expressed as mean \pm standard deviation (SD). One-way ANOVA was employed to determine statistical differences among multiple groups, and t-test was employed to determine the same between two groups. P-values $<0.05$ were considered statistically significant $(\mathrm{P}<0.05, \mathrm{P}<0.01)$. Histological scores were analyzed by the Mann-Whitney U test.

\section{Results}

DSS-induced colitis model. In order to establish DSS-induced colitis, mice were administered 5\% DSS in the drinking fluid for 6 days and weighed every day. DSS-treated mice drastically lost weight from the 4th day after initiation of the medication, compared with that of untreated animals (Fig. 1A). In addition, DSS-treated mice also had remarkably shortened colon (Fig. 1B). these results indicated the successful construction of mice colitis.

Influences of B.s-Dia oral administration on mouse hemafecia and body weight. To evaluate the effects of B.s-Dia on colitis, mice were orally administrated $1 \times 10^{9} \mathrm{cfu}$ B.s-Dia every day, followed by adding 5\% DSS in the drinking water for 7 days (Fig. 2A). Though, both B.s and B.s-Dia oral administration observably diminished bloody stools as showed in 


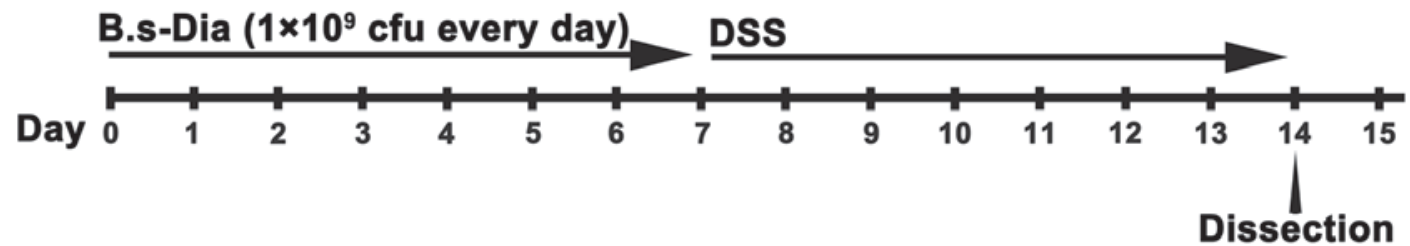

B

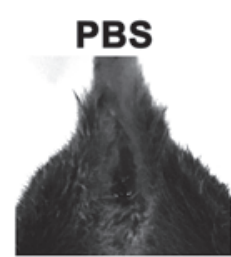

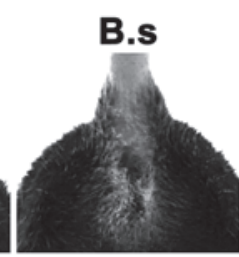

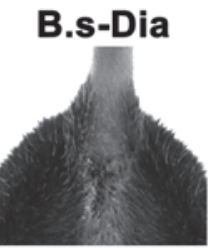

C

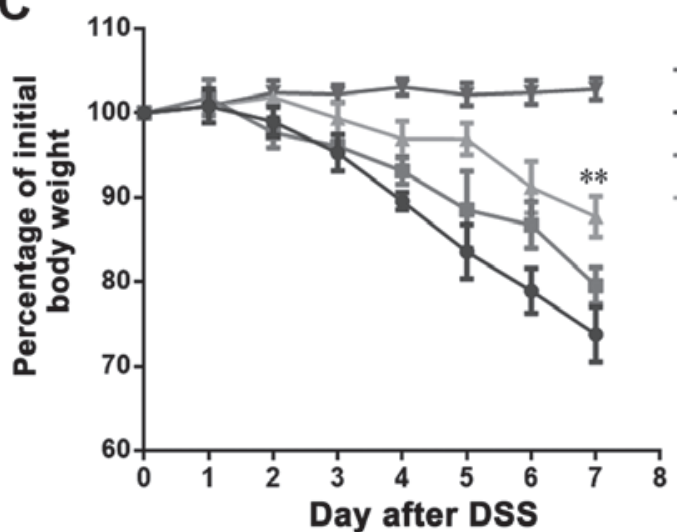

Figure 2. Influences of B.s-Dia oral administration on mouse hemafecia and body weight. (A) The graphical representation of mouse treatment, taken B.s-Dia as example. (B) Anus of mice from different groups were photographed. (C) Mice were administered 5\% DSS in the drinking fluid for 7 days and weighed every day. One representative of three similar independent experiments is shown. Asterisks indicate statistical significance via the one-way ANOVA test $\left({ }^{* *} \mathrm{P}<0.01\right.$ vs. PBS+DSS-treated group. n=7. DSS, dextran sodium sulfate; B.s, Bacillus subtilis; B.s-Dia, 4,4'-diaponeurosporene-producing Bacillus subtilis.
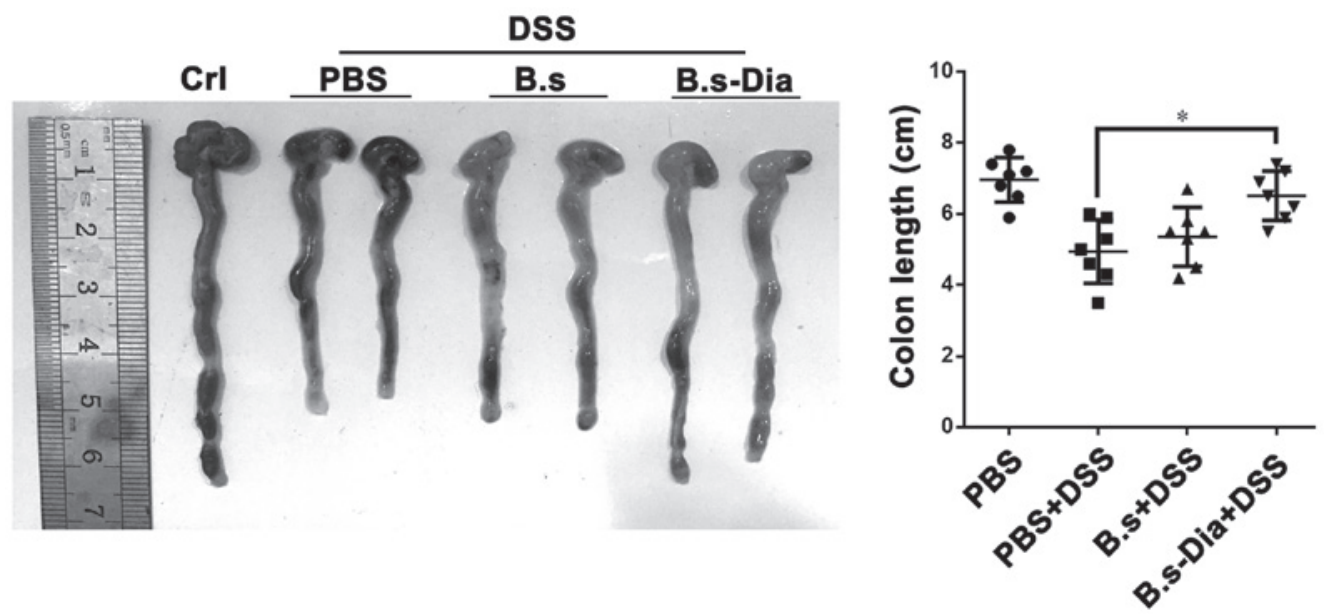

Figure 3. Influences of B.s-Dia oral administration on colon length. The colon of mice from different groups was isolated and placed on a non-absorbent surface and its length was measured with a ruler. One representative of three similar independent experiments is shown. Asterisks indicate statistical significance via the one-way ANOVA test ("P<0.05). n=7. DSS, dextran sodium sulfate; Crl, control; B.s, Bacillus subtilis; B.s-Dia, 4,4'-diaponeurosporene-producing Bacillus subtilis.

Fig. 2B, B.s-Dia was more effective in this process compared with B.s. Importantly, mice in the B.s-Dia group had significantly reduced weight loss than that in the DSS or B.s group on day 7 (Fig. 2C).

Influences of B.s-Dia oral administration on colon length. A shorter colon length and bleeding are considered as a hallmark of experimental colitis $(32,33)$. Our result revealed that oral administration of B.s-Dia significantly increased colon length in experimental colitis, while B.s oral administration had no significantly effect on colon length (Fig. 3). Nevertheless, both B.s and B.s-Dia reduced the severity of bleeding in colon wall.
Influences of B.s-Dia on histological damage induced by DSS. To further evaluate the effects of B.s-Dia on DSS-induced colitis, we performed histopathological examination. As showed in Fig. 4A, the surface epithelium, cryptal glands, mucosa, and submucosa in the normal mice were intact. However, DSS-treated mice showed severe damage of the surface epithelium, bleeding, disruption of the cryptal glands, and thickened submucosal layer. Mice administered B.s showed relatively intact surface epithelium, but the disruption of cryptal glands and infiltration of the inflammatory cells were still observed. However, oral administration of B.s-Dia showed more protective effects and improved DSS-induced pathology (Fig. 4B). 
A

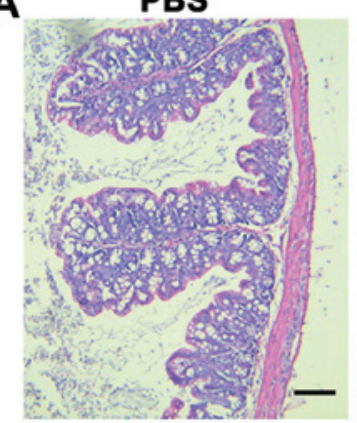

PBS + DSS

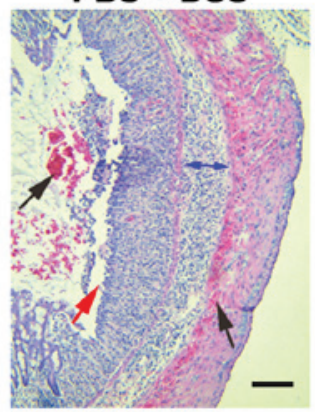

B.S + DSS

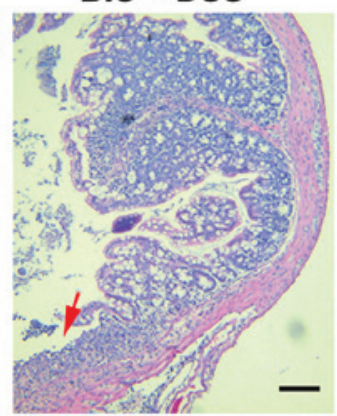

B.s-Dia + DSS

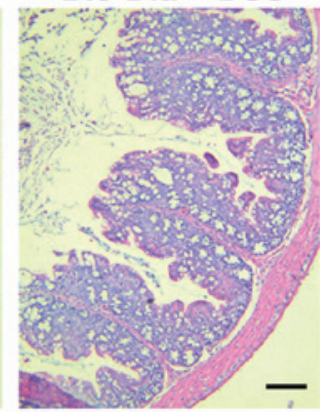

B

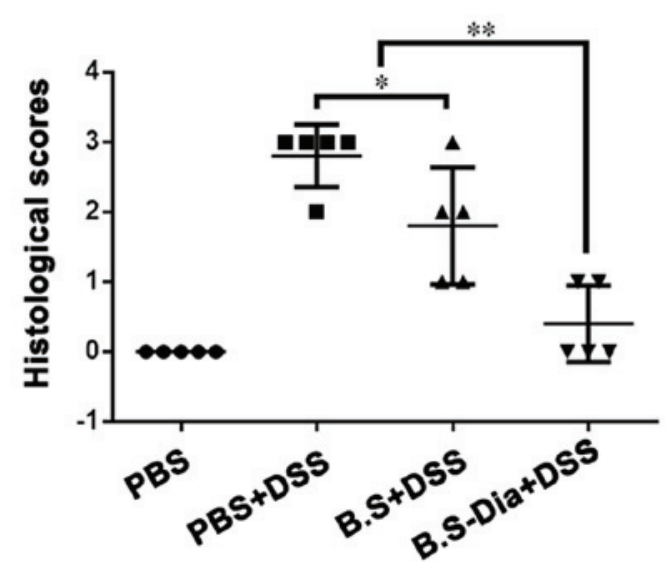

Figure 4. Influences of B.s-Dia on histological damage induced by DSS. (A) The colon of mice from different groups was fixed in $4 \%$ paraformaldehyde, embedded in paraffin and sliced into sections of $5 \mu \mathrm{m}$ thickness, and followed by hematoxylin and eosin staining. bleeding (black arrow), cell detachment (red arrow), thickened submucosal layer (double sided arrow). (B) Colitis was assessed with tissue sections and was scored in a blinded experimental set-up as described in 'Materials and methods'. Histological scores were analyzed by the Mann-Whitney U-test ('P<0.05, ** P $<0.01)$. n=5. Scale bar, 200 nm. DSS, dextran sodium sulfate; B.s, Bacillus subtilis; B.s-Dia, 4,4'-diaponeurosporene-producing Bacillus subtilis.

\section{Discussion}

At present, there is no known cure for human IBD (34). Immunosuppressive therapies, for example with TNF- $\alpha$ antagonists (35), are currently being used as remedies against severe human diseases. Recent investigations have focused on the development of new therapeutic strategies that aim to restore the balance of the gastrointestinal microbiota to reduce or prevent intestinal inflammation. There are growing evidence that probiotic microorganisms might influence disease outcome of IBD in both animal models and humans (36).

The $B$. subtilis species has a long history of safe use and several studies have indicated its ameliorative effect on murine experimental colitis $(15,28)$, In addition, the preventive effect of different $B$. subtilis strains on colitis is not the same (14). because the protective effect of probiotics is related with its antioxidant and anti-inflammatory abilities $(37,38)$. And the severity of colitis induced by the different concentrations of DSS is not the same. Accordingly, $B$. subtilis treated mice still suffer relatively severe colitis.

Carotenoids are a kind of natural pigment with antioxidant properties that prevent oxidative stress. It can also effectively ameliorate symptoms of colitis. Literature shows that $\beta$-carotene treatment ameliorated the severity of UC by modulating various molecular targets (such as nuclear factor- $\mathrm{\kappa B}$, cyclooxygenase-2, interleukin-17) and maintained the gut integrity by increasing the expression of a tight junction protein, occluding, which was decreased in the colon of mice with UC (21). Furthermore, lycopene (39), lutein and zeaxanthin (40) all have effects on colitis. In this study, we showed that 4,4'-diaponeurosporene-producing B. subtilis had an outstanding ability to prevent DSS-induced colitis.

4,4'-Diaponeurosporeneis is also a kind of carotenoids. Our previous study showed that the carotenoid 4,4'-diaponeurosporene modulated hydrogen peroxide-induced oxidative stress in mouse dendritic cells (23), indicating its antioxidant activity. Moreover, it has been reported that the addition of $B$. subtilis to chicken diets can enhance the activity of antioxidant enzymes to scavenge free radicals $(41,42)$. And present study shows that $B$.s-Dia had a more positive effect on DSS-induced colitis, as compared with $B$. subtilis. This result implied the potential beneficial effect of 4,4'-diaponeurosporene on colitis, possibly because of its antioxidant activity. Consequently, Bs-Dia has potential application value for the prevention and treatment of IBD and colitis in clinic.

In brief, the results of the present preliminary study show that Bs-Dia can effectively improve the DSS-induced intestinal mucosal epithelial injury, bleeding, crypts gland rupture and submucosal thickening and other pathological manifestations, greatly alleviate DSS-induced colitis. However, we should note that the mechanism underlies the protection of $B$. subtilis or carotenoids might be complex, and more efforts are needed to illustrate it. Our future studies will aim to investigate the specific factors (intrinsic regulatory mechanisms and epithelial tight junction protein) that B.s-Dia can alleviate colitis, and the dose-dependent effects of B.s-Dia pre-treatment on colitis. 


\section{Acknowledgements}

The present study was supported by 31372465 from the National Science Grant of China and a project funded by the Priority Academic Program Development of Jiangsu Higher Education Institutions (PAPD).

\section{References}

1. Xavier RJ and Podolsky DK: Unravelling the pathogenesis of inflammatory bowel disease. Nature 448: 427-434, 2007.

2. Gismera CS and Aladrén BS: Inflammatory bowel diseases: A disease(s) of modern times? Is incidence still increasing? World J Gastroentero 14: 5491-5498, 2008.

3. Pedersen J, LaCasse EC, Seidelin JB, Coskun M and Nielsen OH: Inhibitors of apoptosis (IAPs) regulate intestinal immunity and inflammatory bowel disease (IBD) inflammation. Trends Mol Med 20: 652-665, 2014.

4. Cleynen I and Vermeire S: Paradoxical inflammation induced by anti-TNF agents in patients with IBD. Nat Rev Gastroenterol Hepatol 9: 496-503, 2012.

5. Naito Y, Takagi T and Yoshikawa T: Molecular fingerprints of neutrophil-dependent oxidative stress in inflammatory bowel disease. J Gastroenterol 42: 787-798, 2007.

6. Roessner A, Kuester D, Malfertheiner P and Schneider-Stock R: Oxidative stress in ulcerative colitis-associated carcinogenesis. Pathol Res Pract 204: 511-524, 2008.

7. Rahimi R, Nikfar S and Abdollahi M: Induction of clinical response and remission of inflammatory bowel disease by use of herbal medicines: A meta-analysis. World J Gastroenterol 19: 5738-5749, 2013.

8. Rahimi R, Mozaffari S and Abdollahi M: On the use of herbal medicines in management of inflammatory bowel diseases: A systematic review of animal and human studies. Dig Dis Sci 54: 471-480, 2009

9. Rahimi R, Shams-Ardekani MR and Abdollahi M: A review of the efficacy of traditional iranian medicine for inflammatory bowel disease. World J Gastroenterol 16: 4504-4514, 2010.

10. Nell S, Suerbaum $S$ and Josenhans $C$ : The impact of the microbiota on the pathogenesis of IBD: lessons from mouse infection models. Nat Rev Microbiol 8: 564-577, 2010.

11. Mimura T, Rizzello F, Helwig U, Poggioli G, Schreiber S, Talbot IC, Nicholls RJ, Gionchetti P, Campieri M and Kamm MA: Once daily high dose probiotic therapy (VSL\#3) for maintaining remission in recurrent or refractory pouchitis. Gut 53: 108-114, 2004.

12. Hong HA, Duc Le H and Cutting SM: The use of bacterial spore formers as probiotics. FEMS Microbiol Rev 29: 813-835, 2005.

13. Sorokulova IB, Pinchuk IV, Denayrolles M, Osipova IG, Huang JM, Cutting SM and Urdaci MC: The safety of two bacillus probiotic strains for human use. Dig Dis Sci 53: 954-963, 2008.

14. Foligné B, Peys E, Vandenkerckhove J, Van Hemel J, Dewulf J, Breton J and Pot B: Spores from two distinct colony types of the strain Bacillus subtilis PB6 substantiate anti-inflammatory probiotic effects in mice. Clin Nutr 31: 987-994, 2012.

15. Gong Y, Li H and Li Y: Effects of Bacillus subtilis on epithelial tight junctions of mice with inflammatory bowel disease. J Interferon Cytokine Res 36: 75-85, 2016.

16. Selvam R, Maheswari P, Kavitha P, Ravichandran M, Sas B and Ramchand CN: Effect of Bacillus subtilis PB6, a natural probiotic on colon mucosal inflammation and plasma cytokines levels in inflammatory bowel disease. Indian J Biochem Biophys 46: 79-85, 2009.

17. Chew BP and Park JS: Carotenoid action on the immune response. J Nutr 134: 257S-261S, 2004.

18. Nair S, Norkus EP, Hertan H and Pitchumoni CS: Micronutrient antioxidants in gastric mucosa and serum in patients with gastritis and gastric ulcer: Does helicobacter pylori infection affect the mucosal levels? J Clin Gastroenterol 30: 381-385, 2000.

19. Rumi G Jr, Szabó I, Vincze A, Matus Z, Tóth G and Mózsik G: Decrease of serum carotenoids in crohn's disease. J Physiology Paris 94: 159-161, 2000.

20. Yasui Y, Hosokawa M, Mikami N, Miyashita $\mathrm{K}$ and Tanaka T: Dietary astaxanthin inhibits colitis and colitis-associated colon carcinogenesis in mice via modulation of the inflammatory cytokines. Chem Biol Interact 193: 79-87, 2011.

21. Trivedi PP and Jena GB: Mechanistic insight into beta-carotene-mediated protection against ulcerative colitis-associated local and systemic damage in mice. Eur J Nutr 54: 639-652, 2015.

22. Bhullar KS, Parmar I and Dhillon GS: Gastrointestinal diseases and curcumin: Developments and challenges. Curr Res Nutr Food Sci 2: 111-113, 2014.
23. Liu H, Xu W, Chang X, Qin T, Yin Y and Yang Q: 4,4'-diaponeurosporene, a C30 carotenoid, effectively activates dendritic cells via CD36 and NF- $\kappa$ B signaling in a ROS independent manner. Oncotarget 7: 40978-40991, 2016.

24. Dong H, Huang Y, Yao S, Liang B, Long Y, Xie Y, Mai J, Gong S and Zhou Z: The recombinant fusion protein of cholera toxin B and neutrophil-activating protein expressed on Bacillus subtilis spore surface suppresses allergic inflammation in mice. Appl Microbiol Biotechnol 101: 5819-5829, 2017.

25. Pazzini CA, Pereira LJ, da Silva TA, Montalvany-Antonucci CC, Macari S, Marques LS and de Paiva SM: Probiotic consumption decreases the number of osteoclasts during orthodontic movement in mice. Arch Oral Biol 79: 30-34, 2017.

26. Starosila D, Rybalko S, Varbanetz L, Ivanskaya N and Sorokulova I: Anti-influenza activity of a Bacillus subtilis probiotic strain. Antimicrob Agents Chemother 61: e00539-e00617, 2017.

27. Wu Z, Tang Z, Shang M, Zhao L, Zhou L, Kong X, Lin Z, Sun H, Chen T, Xu J, et al: Comparative analysis of immune effects in mice model: Clonorchis sinensis cysteine protease generated from recombinant Escherichia coli and Bacillus subtilis spores. Parasitol Res 116: 1811-1822, 2017.

28. Zhang HL, Li WS, Xu DN, Zheng WW, Liu Y, Chen J, Qiu ZB, Dorfman RG, Zhang J and Liu J: Mucosa-reparing and microbiota-balancing therapeutic effect of Bacillus subtilis alleviates dextrate sulfate sodium-induced ulcerative colitis in mice. Exp Ther Med 12: 2554-2562, 2016.

29. Song WJ, Li Q, Ryu MO, Ahn JO, Ha Bhang D, Chan Jung Y and Youn HY: TSG-6 Secreted by human adipose tissue-derived mesenchymal stem cells ameliorates DSS-induced colitis by inducing M2 macrophage polarization in mice. Sci Rep 7: 5187, 2017.

30. Wang B and Wu C: Dietary soy isoflavones alleviate dextran sulfate sodium-induced inflammation and oxidative stress in mice. Exp Ther Med 14: 276-282, 2017.

31. Mazmanian SK, Round JL and Kasper DL: A microbial symbiosis factor prevents intestinal inflammatory disease. Nature 453: 620-625, 2008.

32. Chassaing B, Aitken JD, Malleshappa M and Vijay-Kumar M: Dextran sulfate sodium (DSS)-induced colitis in mice. Curr Protoc Immunol 15: 25, 2014.

33. Ko SJ, Bu Y, Bae J, Bang YM, Kim J, Lee H, Beom-Joon L, Hyun YH and Park JW: Protective effect of Laminaria japonica with probiotics on murine colitis. Mediators Inflamm 2014: 417814, 2014.

34. Prisciandaro L, Geier M, Butler R, Cummins A and Howarth G: Probiotics and their derivatives as treatments for inflammatory bowel disease. Inflamm Bowel Dis 15: 1906-1914, 2009.

35. Feagan BG, Sandborn WJ, Baker JP, Cominelli F, Sutherland LR, Elson CD, Salberg B, Archambault A, Bernstein CA, Lichtenstein GR, et al: A randomized, double-blind, placebo-controlled, multi-center trial of the engineered human anti body to TNF (CDP571) for steroid sparing and maintenance of remission in patients with steroid-dependent Crohn's disease. Gastroenterology 118: A655, 2000.

36. Damaskos D and Kolios G: Probiotics and prebiotics in inflammatory bowel disease: Microflora 'on the scope'. Br J Clin Pharmacol 65: 453-467, 2008.

37. Ghouri YA, Richards DM, Rahimi EF, Krill JT, Jelinek KA and DuPont AW: Systematic review of randomized controlled trials of probiotics, prebiotics and synbiotics in inflammatory bowel disease. Clin Exp Gastroenterol 7: 473-487, 2014.

38. Paynich ML, Jones-Burrage SE and Knight KL: Exopolysaccharide from Bacillus subtilis induces anti-inflammatory M2 macrophages that prevent T cell-mediated disease. J Immunol 198: 2689-2698, 2017.

39. Gul Baykalir B, Aksit D, Dogru MS, Hanım Yay A, Aksit H, Seyrek K and Attesahin A: Lycopene ameliorates experimental colitis in rats via reducing apoptosis and oxidative stress. Int J Vitam Nutr Res: 1-9, 2017.

40. Glabska D, Guzek D, Zakrzewska P, Wlodarek D and Lech G: Lycopene, lutein and zeaxanthin may reduce faecal blood, mucus and pus but not abdominal pain in individuals with ulcerative colitis. Nutrients 8: E613, 2016.

41. Bai K, Huang Q, Zhang J,He J, Zhang L and Wang T: Supplemental effects of probiotic Bacillus subtilis fmbJ on growth performance, antioxidant capacity and meat quality of broiler chickens. Poult Sci 96: 74-82, 2017.

42. Bai WK, Zhang FJ, He TJ, Su PW, Ying XZ, Zhang LL and Wang T: Dietary probiotic Bacillus subtilis strain fmbj increases antioxidant capacity and oxidative stability of chicken breast meat during storage. PLoS One 11: e0167339, 2016. 